\title{
OPEN Directional excitation of surface plasmon using multi-mode interference in an aperture
}

\author{
M. Z. Alam ${ }^{1 凶}$, Z. Yang ${ }^{2}{ }^{2}$, M. Sheik-Bahae' ${ }^{2}$, J. S. Aitchison ${ }^{3} \&$ M. Mojahedi ${ }^{3}$
}

Plasmonics is a promising technology that can find many applications in nanophotonics and biosensing. Local excitation of surface plasmons with high directionality is required for many of these applications. We demonstrate that by controlling the interference of light in a metal slot with the adjustment of the angle of incidence, it is possible to achieve highly directional surface plasmon excitation. Our numerical analysis of the structure showing a strong directionality of excited surface plasmon is confirmed by near field scanning measurements. The proposed structure can be useful for many applications including excitation of plasmonic waveguides, nanolithography, and optical sensing. To illustrate its usefulness, we experimentally demonstrate that it can be used for highly directional excitation of a dielectric loaded plasmonic waveguide. We also propose a simple structure for surface plasmon interference lithography capable of providing high image contrast using this scheme.

Surface plasmon (SP) - the collective oscillations of electrons at the interface between a metal and dielectric can be useful for a wide range of applications ranging from communication to biosensing ${ }^{1-4}$. The most commonly used methods to excite SP are by the use of prisms, or gratings. These schemes are not very compact and introduce a lot of noise from the illumination source, hence they are often not suitable for nanophotonic applications. As an alternative to these traditional approaches, direct illumination through a slot in metal layer has been used as a highly localized source of $\mathrm{SP}^{5}$ - this scheme is simple and noise free. However, the resulting SP radiates away from the hole in every direction, which diminishes its usefulness for many applications. Unidirectional and local excitation of SP has been an active area of research, and many schemes have been proposed to achieve this goal including: gratings, multiple coherent beams, partially filled or asymmetric holes ${ }^{6-22}$. However, these approaches suffer from a number of limitations including complicated geometries and narrow bandwidth. As a result, nonlocal excitation schemes are still commonly used in nanoplasmonics ${ }^{23-31}$. A compact and simple approach which provides highly directional SP excitation will be a key step towards making plasmonics a more useful technology. The improved control of near field that will be available from such a scheme may also lead to many new applications in various areas of nanophotonics.

In this work we present a very simple method for achieving unidirectional SP excitation through a metal slot. The unidirectionality is achieved by controlling the interference of the modes supported by the slot using the angle of incidence of the input light. We verify the effectiveness of this scheme by measuring the near field intensity distribution of the SP around a narrow slot in a continuous metal layer, under oblique backside illumination. The unidirectionality we achieved is much larger than predicted by previous theoretical estimates for oblique backside illumination of metal slots ${ }^{22}$. The proposed scheme extends the flexibility in manipulation of the near field, and can lead to various useful applications. To illustrate this, we experimentally demonstrate coupling of light to a dielectric loaded plasmon waveguide. In addition, we present the concept of a plasmonic nanolithography scheme, which is simple to implement, and provides high image contrast.

\section{Principle of operation}

When a slot in a metal film is illuminated by light from the back side, the light transmission is mediated by the modes excited in the slot, which acts as a waveguide ${ }^{32}$. The amount of transmitted power and the modes excited on the exit side of the slot depend on the efficiency of excitation of various modes within the slot and their mutual interactions. We aim to use this phenomenon to achieve unidirectional excitation of SP. To achieve an insight into the various possibilities, we have analyzed the structure shown in Fig. 1a. The structure consists of a slot of

${ }^{1}$ Department of Electrical and Computer Engineering, Queen's University, Kingston K7L 3N9, Canada. ${ }^{2}$ Department of Physics and Astronomy, University of New Mexico, Albuquerque, NM 87131, USA. ${ }^{3}$ Department of Electrical and Computer Engineering, University of Toronto, Toronto M5S 3G4, Canada. ${ }^{\circledR}$ email: m.alam@queensu.ca 



Figure 1. (a) Schematic representation of the single metal slot. (b) Power density profile for a single mode slot $(\mathrm{w}=300 \mathrm{~nm})$. (c) and (d) Power density profiles for a multi-mode slot $(\mathrm{w}=500 \mathrm{~nm})$ when the two supported modes are in phase $\{(\mathbf{c})\}$ and $180^{\circ}$ out of phase $\{(\mathbf{d})\}$ at the bottom of the slot. Gold film thickness (t) is $200 \mathrm{~nm}$.
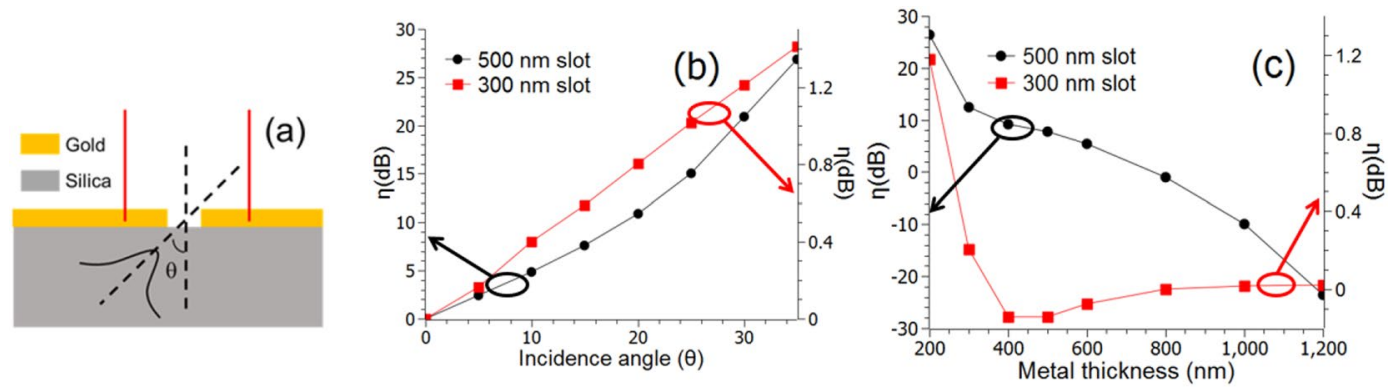

Figure 2. (a) Schematic diagram showing the simulation set up. (b) and (c) Enhancement of unidirectionality as a function of incidence angle and metal film thickness for single mode $(w=300 \mathrm{~nm})$ and multimode $(w=500 \mathrm{~nm})$ slots. Gold thickness for $(\mathbf{b})$ is $200 \mathrm{~nm}$, and the angle of incidence for $(\mathbf{c})$ is $30^{\circ}$.

width $w$, in a gold film of thickness $t$. All numerical results for this work were obtained using Lumerical FDTD with a wavelength of $920 \mathrm{~nm}$ in all cases. The material properties for gold and silica are taken from ${ }^{33,34}$. When the slot is very narrow, e.g. $w=300 \mathrm{~nm}$, only the first-order mode in the metal-dielectric-metal (MIM) waveguide is supported. This mode propagates through the slot and at the end of the slot either couples into the SP modes or radiates into the free space above the metal. The power distribution for this case is shown in Fig. 1b. For a wider slot, multiple modes are supported. As an example, for $w=500 \mathrm{~nm}$, the slot supports two modes. The direction of power flow at the exit of the slot in this case will depend on the relative amplitude and phase of the two modes. To illustrate this, we have plotted the power density profiles for the cases when the two modes are excited with equal amplitude and are either in phase (Fig. 1c) or $180^{\circ}$ out of phase (Fig. 1d). Lumerical mode source has been used in these simulations to properly excite the modes for the analysis reported in Fig. 1. In a physical implementation, the mode excitation can be controlled by a number of ways including multiple beams ${ }^{18}$ or controlling the angle of excitation (proposed in this work). As can be seen from the figures, the interactions of the modes can result in highly directional power flow in the half space above the metal surface. An illustration of this principle for unidirectional SP excitation can be found in ${ }^{18}$. Unlike ${ }^{18}$ where multiple coherent beams were used to properly excite the required modes, we propose to achieve this by adjusting the angle of incidence of a single laser beam. The simplicity of our proposal can be useful for many nanophotonic applications.

The excitation efficiency of different modes and their interaction in the slot are affected by a number of parameters including the angle of incidence, geometry of slot, and metal thickness. Therefore, it is possible to adjust the direction of power flow by controlling any of these parameters. To illustrate this, we study the effects of two parameters, i.e., angle of incidence and metal film thickness on the directionality of the SP. The simulation setup is shown in Fig. 2a. The structure is excited using a Gaussian beam from underneath at an angle $\theta$ with respect to the normal to the metal-silica interface, and the fields are recorded in simulation at equal distance on the two sides of the slot marked by the red lines. Lumerical FDTD's built in mode expansion monitor is then used to decompose the recorded field into various modes to calculate the fraction of the power converted to SP. To quantify the directionality of the power flow, we define a unidirectionality factor $(\eta)$ as

$$
\eta=\frac{S P_{\text {right }}}{S P_{\text {left }}}
$$

Here $S P_{\text {right }}$ and $S P_{\text {left }}$ are SP generated on the right and left sides of the slot. Figure $2 \mathrm{~b}$ shows the values of $\eta$ as a function of incident angle for both single mode $(w=300 \mathrm{~nm})$ and multimode $(w=500 \mathrm{~nm})$ slots. From Fig. 2b the unidirectionality factor ranges from 0 to $1.4 \mathrm{~dB}$ over the range of angles plotted for the single mode slot. In contrast, $\eta$ can be very large, for a multimode slot, over the same range of angles. Figure $2 c$ shows the directionality as a function of metal thickness at a constant incident angle $\left(\theta=30^{\circ}\right)$ for both single and multimode slots. It is clear that $\eta$ is not significantly affected by the metal thickness for a single mode slot but varies widely for a 

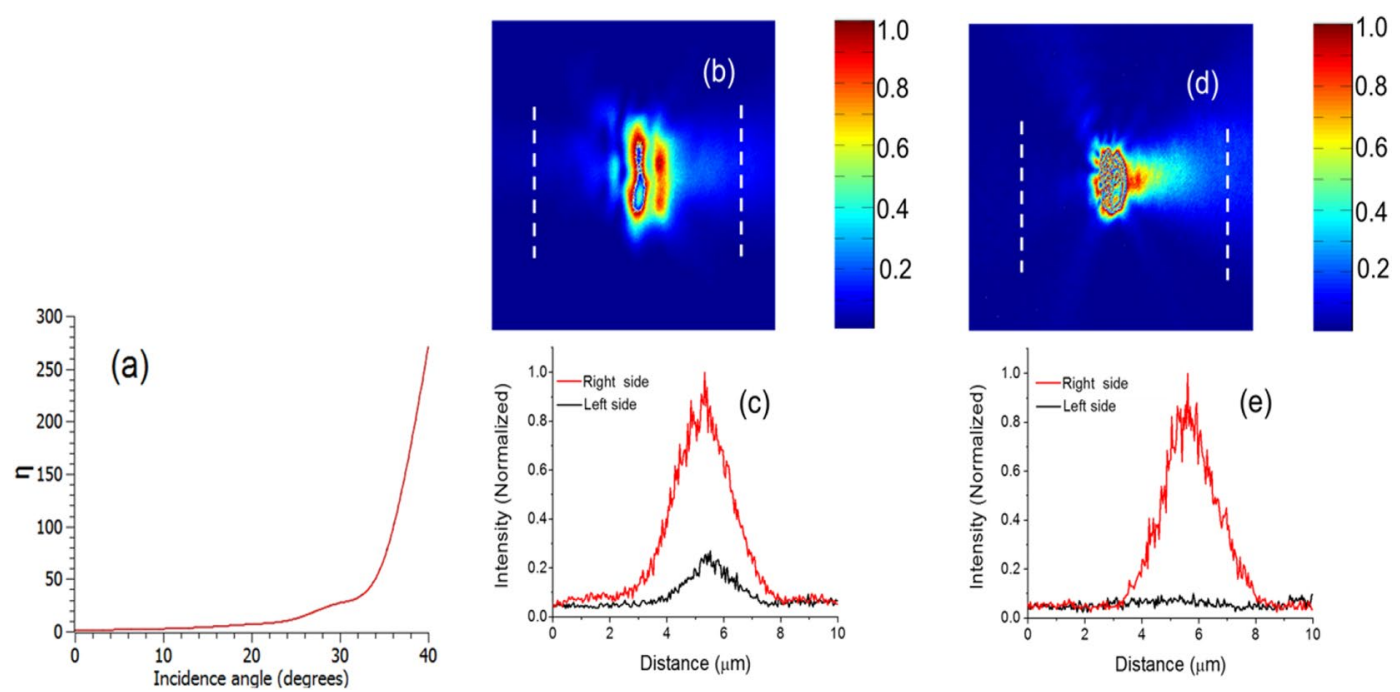

Figure 3. (a) FDTD simulation results for unidirectionality factor $(\eta)$ as a function of the beam tilt for a rectangular hole of dimensions $2 \mu \mathrm{m} \times 0.5 \mu \mathrm{m}$. (b) and (c) Near field measurement results for $\theta=15^{\circ}$. (d) and (e) Near field measurement results for $\theta=42^{\circ}$.

multimode slot. These results can be explained from the physical picture presented in Fig. 1c,d. As the two modes having different effective mode indices propagate through the slot, the relative phase between them changes, and as a result, the power distribution at the exit of the slot also changes. Therefore, it is expected that the metal film thickness has a significant effect on the enhancement in the case of wide slots. At this point, it is relevant to consider the discussion $\mathrm{in}^{22}$, where the authors have concluded that for an oblique incidence the metal film acts as a geometrical shadow. While such a shadowing may be the dominant cause of unidirectionality in the case of single mode slot, it fails to explain the behavior observed in the multimode case. From Fig. $2 \mathrm{c}$ it can be observed that high level of unidirectionality can be achieved toward the right side or the left side, even when the beam is only tilted to the right. Also $\eta$ varies strongly with metal thickness in the multimode case but remains relatively unchanged in the single mode case. These trends are difficult to explain using the notion of geometrical shadowing alone but can be easily explained with the concept of multimode interference. Guided by this concept we have, for the first time, observed that very large value of $\eta$ can be achieved by simple adjustment of beam tilt without the need for any complex geometry employed in many previously reported structures.

The example of the two-dimensional structure discussed above illustrates that excitation of various modes to achieve unidirectional excitation is possible by simply adjusting the angle of incidence of incident light. The principle is very general, and can be extended to three dimensions, which are more relevant for practical applications. To verify this, we fabricated a series of isolated holes in a $200 \mathrm{~nm}$ thick gold film and carried out near field measurements. Details of fabrication and measurements are provided at the end of the paper. Here we present results for a $2 \mu \mathrm{m} \times 0.5 \mu \mathrm{m}$ rectangular hole. Figure 3a shows $\eta$ predicted by FDTD as a function of angle of incidence for this hole. Figure $3 \mathrm{~b}$ shows the measured near-field profile for $\theta=15^{\circ}$. For ease of visualization in Fig. $3 \mathrm{c}$ we have plotted the intensity on both sides of the holes along the dashed lines in Fig. $3 \mathrm{~b}$. The measured $\eta$ for $\theta=15^{\circ}$ is approximately 4, compared to 4.1 from FDTD simulation. Figure 3d,e are near field measurement results for $\theta=42^{\circ}$. The signal in the left side of the hole in this case is very close to the noise level, and as a result it is not possible to measure the exact value of $\eta$ from these measurements. However, these results confirm the predictions from FDTD that the directionality of SP excitation is much higher for $\theta=42^{\circ}$ than compared to $\theta=15^{\circ}$.

\section{Applications of the proposed excitation scheme}

The proposed SP excitation scheme is simple to implement and can provide very high degree of unidirectionality. In the following, we present two examples to illustrate its usefulness: local excitation of plasmonic waveguides and surface plasmon nanolithography.

Local excitation of plasmonic waveguides. To illustrate the usefulness of the proposed excitation scheme for plasmonic waveguides, we fabricated and tested the structure shown in Fig. 4a. It consists of a PMMA waveguide only on one side of the hole. This allows us to compare the light intensity at two sides of the hole and estimate unidirectionality. Figure $4 \mathrm{~b}$ shows the fabricated structure. It consists of a circular hole with $800 \mathrm{~nm}$ diameter milled in $200 \mathrm{~nm}$ thick gold film. The hole is adjacent to a $5 \mu \mathrm{m}$ long and $300 \mathrm{~nm}$ thick PMMA line. Figure 4c shows the near field profile calculated by Lumerical FDTD for light of $920 \mathrm{~nm}$ wavelength propagating along the $\mathrm{z}$ axis and illuminating the hole from beneath the metal surface. Electric field for the illumination is aligned along the $y$ direction. Figure $5 \mathrm{~d}$ shows the measured near field profile on the metal surface for the same illumination condition. Because of the finite size of the NSOM tip (200 nm diameter), the optical resolution is moderate as expected ${ }^{35}$. The good agreement between the FDTD results and the NSOM measurement is evident 

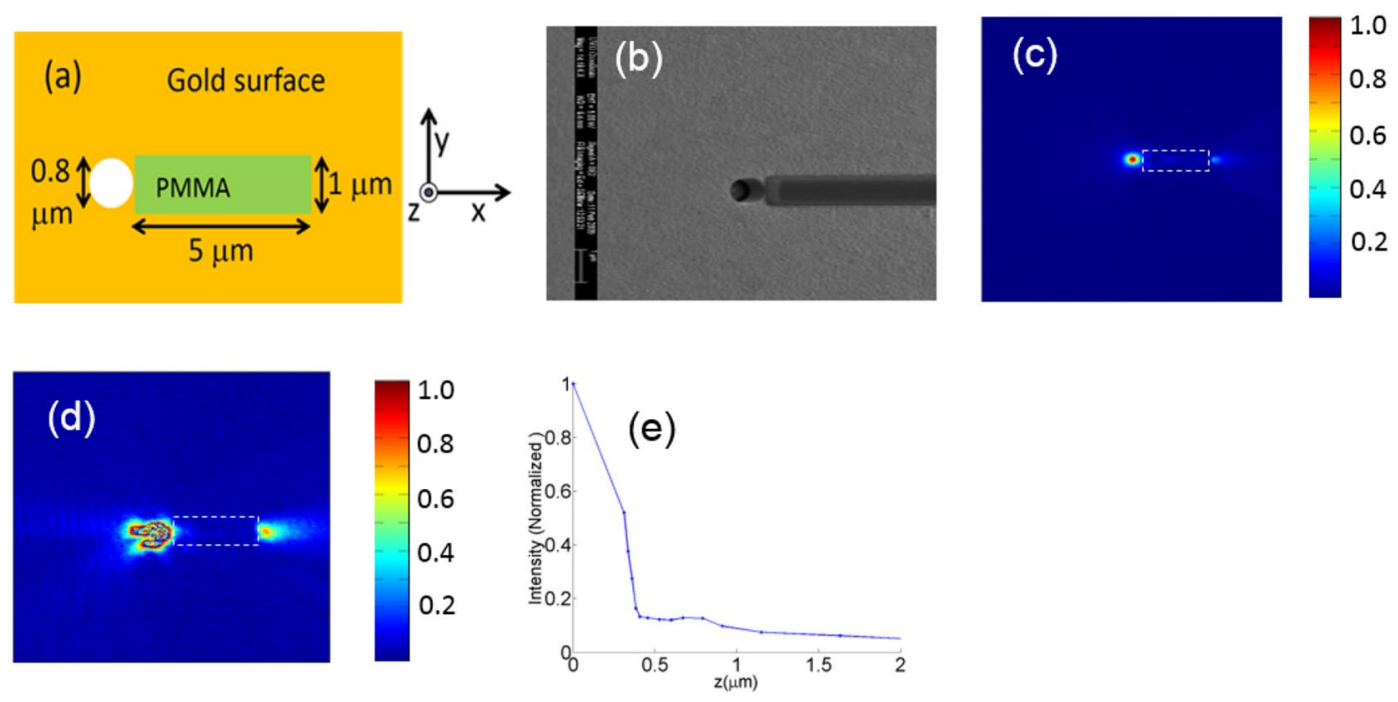

Figure 4. (a) Proposed structure. (b) Fabricated sample. (c) Intensity above the sample surface calculated using FDTD. (d) NSOM measurement results. (e) Intensity measured along the vertical direction ( $z$-axis) after the end of the waveguide.
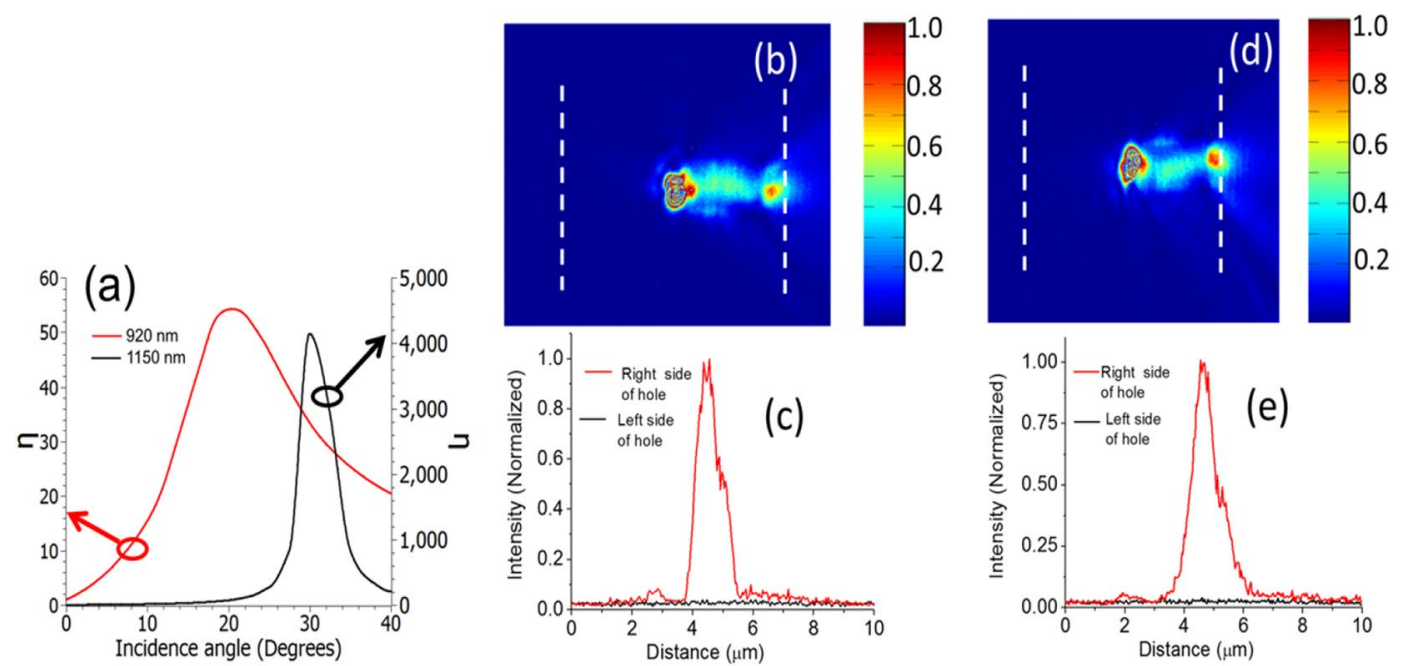

Figure 5. (a) Unidirectionality for various tilt angles for the structure shown in Fig. 4a. (b) and (c) Near field measurement results for $\theta=22^{\circ}$. (d) and (e) Near field measurement results for $\theta=37^{\circ}$.

from Fig. 4c,d. To verify that light exiting the hole is indeed converted to SP, we measured the light intensity along the vertical $(z)$ direction. The field confinement $0.5 \mu \mathrm{m}$ after the end of the waveguide is shown in Fig. 5e. The field has higher confinement at the metal surface, and decays as we move away from metal surface indicating that the PMMA waveguide is successful in converting power exiting the slot into the SP mode. In addition to the simulation and experimental results presented in this section, we also present a simplified theoretical model in the Supplementary Information section, which provides insight into the effect of dielectric film thickness on the surface plasmon excitation efficiency.

The unidirectionality measured for normal incidence case is approximately 8.7 , which is comparable with previous reports ${ }^{31}$. Higher level of unidirectionality would be required in many practical applications. This can be achieved by properly choosing the angle of incidence and having the PMMA guide, which will combine two effects to enhance unidirectionality: (a) sending most power to one side at the exist of the slot (by controlling the mode excitation by suitable choice of angle of incidence) and (b) by coupling light at the exit of the slot to SP (by proper choice of PMMA height). Figure 5a shows $\eta$ as a function of tilt angle calculated using FDTD for the structure shown in Fig. $4 \mathrm{a}$ for two different wavelengths $(920 \mathrm{~nm}$ and $1150 \mathrm{~nm})$. These results illustrate that high unidirectionality can be achieved using this structure over a broad wavelength range. Figure $5 b, c$ show the measured near field profiles at $920 \mathrm{~nm}$ wavelength for $22^{\circ}$ angle of incidence. Figure 5d,e show the near-field profiles for $37^{\circ}$ for the same sample. As expected from simulations, the SP excitation is highly unidirectional 

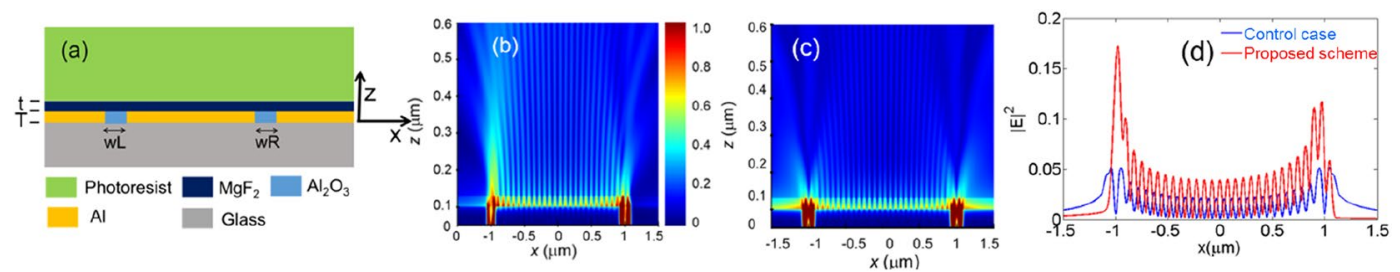

Figure 6. (a) Schematic of the proposed SPIL scheme. Dimensions are $\mathrm{t}=30 \mathrm{~nm}, \mathrm{~T}=100 \mathrm{~nm}, \mathrm{wL}=105 \mathrm{~nm}$, $\mathrm{wR}=140 \mathrm{~nm} . \mathrm{z}=0$ is located at the metal- $\mathrm{MgF}_{2}$ interface, and $\mathrm{x}=0$ is the midpoint between the two slots. (b) and (c) FDTD results for square of electric field amplitude $\left(|\mathrm{E}|^{2}\right)$ for proposed scheme and control case $(\mathbf{d})(|\mathrm{E}|)^{2}$ across a line $50 \mathrm{~nm}$ above the metal for tilted excitation ( $37^{\circ}$ from $\mathrm{z}$ axis) shown by the red line. For comparison, $|\mathrm{E}|^{2}$ for the control case is shown by blue line.

for both angles. The low power level on the left side of the hole makes it challenging to measure $\eta$, but the high levels of directionality for both angles are evident from these measurements.

Surface plasmon interference lithography. Surface plasmon interference lithography (SPIL) is an emerging lithographic method capable of overcoming the diffraction limit of conventional photolithography ${ }^{36-38}$. The most important figure of merit of SPIL is the image contrast. Many previously reported SPIL schemes fail to achieve high image contrast, or achieve this by utilizing structures, which are challenging to fabricate. Previously we explained that the direction of SP can be controlled by adjusting geometrical parameter and angle of illumination. Here we use this principle to design a SPIL scheme for deep ultraviolet lithography to overcome some of the limitations of previous proposals. As shown in Fig. 6a, the proposed structure consists of two slots in a $100 \mathrm{~nm}$ thick aluminum film. The slots are filled with alumina, and the entire structure is coated with a $30 \mathrm{~nm}$ thick magnesium fluoride $\left(\mathrm{MgF}_{2}\right)$ layer. The presence of the $\mathrm{MgF}_{2}$ film leads to large light intensity near the metal surface similar to conductor gap mode ${ }^{39}$ and provides high light intensity near the metal surface. We assume that a semi-infinite photoresist having refractive index 1.7 fills the upper half space above the mask. The width of the left and right slots are $105 \mathrm{~nm}$ and $140 \mathrm{~nm}$ respectively. These dimensions are chosen to ensure that, under oblique illumination, the SPs exiting the holes will propagate towards each other, and create an interference pattern between the slots. Figure $6 \mathrm{~b}$ shows the square of the electric field amplitude $\left(|\mathrm{E}|^{2}\right)$ across the structure when it is illuminated from the bottom at $37^{\circ}$ with respect to normal to the metal surface at $260 \mathrm{~nm}$ wavelength. The SP generated by the left and right hole propagates toward each otherand form a standing wave pattern between the slots. Figure $6 \mathrm{c}$ shows $|\mathrm{E}|^{2}$ for a control case. The structure for the control case is identical to that shown in Fig. $6 \mathrm{a}$ with the exception that both slots are $140 \mathrm{~nm}$ wide. The illumination is normal to the surface in that case. Figure $6 \mathrm{~d}$ shows $|\mathrm{E}|^{2}$ at a distance of $50 \mathrm{~nm}$ above the metal film for the proposed structure (red line). For comparison $|\mathrm{E}|^{2}$ for the control case for the same location is also shown in the same figure (blue line). The half pitch of the interference pattern is $36 \mathrm{~nm}$-much smaller than achievable by conventional photolithography. As shown in Fig. 6d, the proposed scheme provides larger contrast in the region between the two slots (which we will call "slot region") than the control case, which will make it useful for a wider range of photolithography processes. For the control case, the field intensity is significant outside the slot region. This field may result in formation of unwanted interference pattern outside the slot region, if any surface feature is present nearby. In contrast for the proposed scheme, the electric field strongly decays outside the slot region, and the chance of interference patterns forming outside the slot region is greatly reduced. The simplicity of the proposed scheme, its ability to provide large contrast, and highly localized field profile will extend the applicability of SPIL for nanofabrication.

\section{Conclusions}

We have proposed and experimentally demonstrated that oblique backside illumination can be a simple method for achieving unidirectional SP excitation. We show that light can be coupled to a dielectric loaded plasmon waveguide with high directionality by using this simple geometry. The directionality we achieved is better than many previous proposals that uses a more complicated geometry. Because of the limitations of previously reported local SP excitation schemes, far field excitation is still commonly used in nanoplasmonics ${ }^{24-29}$. We believe the simplicity of this scheme will make it a very suitable choice of excitation for many widely used plasmonic waveguides ${ }^{40}$. We also present the design of a simple scheme of surface plasmon interference lithography capable of providing very large image contrast. We believe the simplicity, and the effectiveness of the proposed scheme will make it a widely used method for many applications including plasmonic sensing ${ }^{41}$, read/write operation in optical storage medium $^{42}$, and controlling the near field interactions in plasmonic random metasurfaces ${ }^{43}$.

\section{Methods}

Sample preparation. The gold films were supplied by Ssens Bv. The sample consists of a $200 \mathrm{~nm}$ thick gold film with a thin titanium adhesion layer deposited on a glass substrate. A number of isolated holes were milled on the gold surface using a focused ion beam. The structure shown in Fig. $4 \mathrm{~b}$ was fabricated as follows. Holes were milled with a focused ion beam in a $200 \mathrm{~nm}$ thick gold film on a glass substrate. For the fabrication of the PMMA waveguide, 950 PMMA A3 was spun on the sample and the pattern was defined using a Vistec $5000+$ electron beam lithography system and developed using a MIBK:IPA developer solution. 
Measurement set up. $\quad 300 \mathrm{~mW}$ input power from a Ti-Sapphire laser at $920 \mathrm{~nm}$ wavelength was coupled through the hole by a series of optical components. In the beam path two polarizers were used. By keeping the second polarizer at a fixed angle, and rotating the first one, we controlled the power incident on the sample, while linear polarization is maintained. To avoid over heating of sample, and possible damage to the PMMA waveguides we use a relatively large spot size (diameter $7 \mathrm{~mm}$ ). The angle of incidence of light to the sample is controlled by using a tilted mirror. The spot size of the illumination is approximately $100 \mu \mathrm{m}$. The near field images were recorded using Multiview-100 near field scanning optical microscope working in the collection mode to measure the near field on top of the metal surface. Each point in the NSOM images is collected with $10 \mathrm{~ms}$ average time. Each frame is 256 by 256 pixels.

Received: 28 June 2018; Accepted: 19 November 2020

Published online: 04 February 2021

\section{References}

1. Barnes, W. L., Dereux, A. \& Ebbesen, T. W. Surface plasmon subwavelength optics. Nature 424, 824-830 (2003).

2. Atwater, H. A. The promise of plasmonics. Sci. Am. 17, 56-63 (2007).

3. Tong, L., Wei, H., Zhang, S. \& Xu, H. Recent advances in plasmonic sensors. Sensors 14, 7959-7973 (2014).

4. Ozbay, E. Plasmonics: merging photonics and electronics at nanoscale dimensions. Science 311, 189-193 (2006).

5. Yin, L. et al. Surface plasmons at single nanoholes in Au films. Appl. Phys. Lett. 85, 467-469 (2004).

6. Dewanjee, A., Alam, M. Z., Aitchison, J. S. \& Mojahedi, M. Highly efficient excitation of surface plasmons using a Si gable tip. Laser Photonics Rev. 11, 1700009 (2017).

7. Jiang, Q. et al. Directional and singular surface plasmon generation in chiral and achiral nanostructures demonstrated by leakage radiation microscopy. ACS Photonics 3, 1116-1124 (2016).

8. Guo, Q., Zhnag, C. \& Hu, X. A spiral plasmonic lens with directional excitation of surface plasmons. Sci. Rep. 6, 32345 (2016).

9. Yao, W. et al. Efficient directional excitation of surface plasmons by a single-element nanoantenna. Nano Lett. 15, 3115-3121 (2015).

10. Huang, W., Yang, J., Xiao, X. \& Zhang, J. Surface plasmon polariton unidirectional nano-launcher based on the strong coupling effects in an asymmetric optical slot nanoantenna pair. Plasmonics 10, 1551-1556 (2015).

11. Yang, J. et al. Broadband surface plasmon polariton directional coupling via asymmetric optical slot nanoantenna pair. Nano Lett. 14, 704-709 (2014).

12. Fortnuno, F. J. R. et al. Near-field interference for the unidirectional excitation of electromagnetic guided modes. Science 19, 328-330 (2013).

13. Huang, X. L. \& Brongersma, M. L. Compact aperiodic metallic groove arrays for unidirectional launching of surface plasmons. Nano Lett. 13, 5420-5424 (2013).

14. Gao, W. et al. Excitation and active control of propagating surface plasmon polaritons in graphene. Nano Lett. 13, 3698-3702 (2013).

15. Li, D. et al. Unidirectional surface plasmon-polariton excitation by a compact slot partially filled with dielectric. Opt. Exp. 21, 5949-5956 (2013)

16. Liu, Y. et al. Compact magnetic antennas for directional excitation of surface plasmons. Nano Lett. 12, 4853-4858 (2012).

17. Baron, A. et al. Compact antenna for efficient and unidirectional launching and decoupling of surface plasmons. Nano Lett. 11, 4207-4212 (2011).

18. Raghunathan, S. B. et al. Plasmon switching: observation of dynamic surface plasmon steering by selective mode excitation in a sub-wavelength slit. Opt. Exp. 20, 15326 (2012).

19. Chen, J., Li, Z., Yue, S. \& Gong, Q. Efficient unidirectional generation of surface plasmon polaritons with asymmetric singlenanoslit. Appl. Phys. Lett. 97, 041113 (2010).

20. Tejeira, F. L. et al. Efficient unidirectional nanoslit couplers for surface plasmons. Nat. Phys. 3, 324-328 (2007).

21. Jin, J. et al. Polarization controlled unidirectional excitation of surface plasmon polaritons utilizing catenary apertures. Nanoscale 11, 3952 (2019).

22. Kim, H. \& Lee, B. Unidirectional surface plasmon polariton excitation on single slit with oblique backside illumination. Plasmonics 4, 153-159 (2009)

23. Seidel, A. et al. Demonstration of laser-fabricated DLSPPW at telecom wavelength. IEEE Photonics J. 4, 652-658 (2010).

24. Oulton, R. F. et al. Plasmon lasers at deep subwavelength scale. Nature 461, 629-632 (2009).

25. Kravets, V. G. et al. Graphene-protected copper and silver plasmonics. Sci. Rep. 4, 5517 (2014).

26. Constant, T. J., Hornett, S. M., Chang, D. E. \& Hendry, E. All-optical generation of surface plasmons in graphene. Nat. Phys. 12, 124-128 (2016).

27. Block, A. et al. Bloch oscillations in plasmonic waveguide arrays. Nat. Commun. 5, 3843 (2014).

28. Ansell, D. et al. Hybrid graphene plasmonic waveguide modulators. Nat. Commun. 6, 8846 (2015).

29. Steinberger, B. et al. Dielectric stripes on gold as surface plasmon waveguides. Appl. Phys. Lett. 88, 094104 (2006).

30. Mehfuz, R., Maqsood, M. W. \& Chau, K. J. Enhancing the efficiency of slit-coupling to surface-plasmon-polaritons via dispersion engineering. Opt. Exp. 18, 18206-18216 (2010)

31. Mehfuz, R., Chowdhury, F. A. \& Chau, K. J. Imaging slit-coupled surface plasmon polaritons using conventional optical microscopy. Opt. Exp. 20, 10526-10537 (2012).

32. Catrysse, P. B. \& Fan, S. Propagating plasmonic mode in nanoscale apertures and its implications for extraordinary transmission. J. Nanophotonics 2, 021790 (2008).

33. Palik, E. D. Handbook of Optical Constants of Solids (Academic Press, Inc., Cambridge, 1985).

34. Johnson, P. B. \& Christy, R. W. Optical constants of noble metals. Phys. Rev. B 6, 4370-4379 (1972).

35. Wei, C., Tsao, P. S. \& Fann, W. Polarization dependence of light intensity distribution near a nanometric aluminum slit. J. Opt. Soc. Am. B 21, 1005-1012 (2004)

36. Dong, J., Liu, J., Kang, G., Xie, J. \& Wang, Y. Pushing the resolution of photolithography down to $15 \mathrm{~nm}$ by surface plasmon interference. Sci. Rep. 4, 5618 (2014).

37. Xu, T. et al. Subwavelength nanolithography based on unidirectional excitation of surface plasmons. J. Opt. A Pure Appl. Opt. 11, 085003 (2009).

38. Yang, X., Zeng, B., Wang, C. \& Luo, X. Breaking the feature sizes down to sub-22 nm by plasmonic interference lithography using dielectric-metal multilayer. Opt. Exp. 24, 21560-21565 (2009). 
39. Avrutsky, I., Soref, R. \& Buchwald, W. Sub-wavelength plasmon modes in a conductor-gap-dielectric system with a nanoscale gap. Opt. Exp. 18, 348-363 (2010).

40. Alam, M. Z., Meier, J., Aitchison, J. S. \& Mojahedi, M. Propagation characteristics of hybrid modes supported by metal-low-high index waveguides and bends. Opt. Exp. 18, 12971-12979 (2010).

41. Liu, N., Tang, M. L., Hentschel, M., Giessen, H. \& Alivisatos, A. P. Nanoantenna-enhanced gas sensing in a single tailored nanofocus. Nat. Mater. 10, 631-636 (2011).

42. Mansuripur, M. et al. Plasmonic nano-structures for optical data storage. Opt. Exp. 17, 14001-14009 (2009).

43. Siddique, R. H., Mertens, J., Holscher, H. \& Vignolini, S. Scalable and controlled self-assembly of aluminum-based random plasmonic metasurfaces. Light Sci. Appl. 6, e17015 (2017).

\section{Acknowledgement}

The work at the University of New Mexico was partially supported by the NSF under Award DMR-1207489.

\section{Author contributions}

M.Z.A. in consulation with J.S.A. and M.M. conceived the ideas reported in the manscript. M.Z.A. carried out the simulation and fabrication. Z.Y. carried out the measurements under the supervision of M-S.B.M.Z.A. wrote the manuscript, and all the authors reviewed and participated in editing the manuscript.

\section{Competing interests}

The authors declare that they have no competing interests.

\section{Additional information}

Supplementary information is available for this paper at https://doi.org/10.1038/s41598-020-78594-7.

Correspondence and requests for materials should be addressed to M.Z.A.

Reprints and permissions information is available at www.nature.com/reprints.

Publisher's note Springer Nature remains neutral with regard to jurisdictional claims in published maps and institutional affiliations.

(c) Open Access This article is licensed under a Creative Commons Attribution 4.0 International

License, which permits use, sharing, adaptation, distribution and reproduction in any medium or format, as long as you give appropriate credit to the original author(s) and the source, provide a link to the Creative Commons licence, and indicate if changes were made. The images or other third party material in this article are included in the article's Creative Commons licence, unless indicated otherwise in a credit line to the material. If material is not included in the article's Creative Commons licence and your intended use is not permitted by statutory regulation or exceeds the permitted use, you will need to obtain permission directly from the copyright holder. To view a copy of this licence, visit http://creativecommons.org/licenses/by/4.0/.

(C) The Author(s) 2021 